\title{
Consciousness Created by Brain Waves
}

\author{
Tapan Das \\ Director, Advanced Science and Technology, Vitesse, 359 Terry Fox Drive, Ottawa, Ontario K2K2E7, Canada
}

\begin{abstract}
Brain waves measured by EEG are created by very complex interactions of large number of neurons. Brainwaves are in five frequency bands-Delta (0.5-4 Hz), Theta (4-8 Hz), Alpha (8-12 Hz), Beta (12-40 Hz), and Gamma (40-100 Hz). As we become more conscious our brainwave frequency increases. Hence, our consciousness is a function of brain wave frequency and is given by brain wave frequency multiplied by consciousness constant Consciere C. This is similar to any other physical constant like Gravitational constant G, Planck Constant h, etc. When our brain is dead, we have no consciousness.
\end{abstract}

Key words: Consciousness, brainwave, consciere, anesthesia.

\section{Introduction}

This article gives a new theory of consciousness as a function of brain wave and consciousness constant Consciere C. As long as brain waves exist we are conscious. When our brain is dead, hence no brain wave, we have no consciousness.

\section{Consciousness and Brain Waves}

At the root of all our thoughts, behaviors and emotions, is the communication between neurons in our brains. Brainwaves are produced by synchronized electrical pulses from masses of neurons communicating with each other. Brain wave is rhythmic or repetitive neural activity in the brain [1]. This interaction between the neurons giving rise to brain wave at a certain frequency is different from the firing frequency of individual neurons. Consciousness is created by brain waves. The major brain wave frequencies are-Delta, Theta, Alpha, Beta, and Gamma.

Delta: frequency range is $0.5-4$ cycles per second $(\mathrm{Hz})$. They are generated in dreamless and deepest meditation.

Theta: frequency range is 4-8 $\mathrm{Hz}$. They are generated in relaxed state.

Alpha: frequency range is $8-12 \mathrm{~Hz}$. They are

Corresponding author: Tapan Das, M.Sc., Ph.D., research fields: neuroscience, electronics and cosmology. generated in mental coordination, calmness and alertness.

Beta: frequency range is $12-40 \mathrm{~Hz}$. They are generated in focused mental activity, problem solving, judgment and decision making.

Gamma: frequency range is $40-100 \mathrm{~Hz}$. They are generated in cognitive functioning, learning and information processing.

Hence, Brain activity is proportional to the brain wave frequency (BWF). Brain activity needs brain energy. Hence, brain energy is proportional to the brain wave frequency. Consciousness is related to brain activity, hence brain energy. Hence, consciousness is proportional to the brain wave frequency [2]. If physical constant of consciousness is Consciere $\mathrm{C}$, then consciousness is $=C$.f, where $C$ is a physical constant. There are many physical constants in science. Some of the most recognizable ones are gravitational constant $G$, Planck's constant $h$, Boltzmann constant $k_{B}$, speed of light in vacuum $c$, etc.

Let us analyze brain waves of a person in problem solving state. In this case, the dominant waves will be in Beta range, but there will be additional waves in Gamma, Alpha and even Theta ranges. Let us call the brain wave frequencies in Beta range as $f_{b 1}, f_{b 2}, f_{b 3}$, $f_{b 4}$ and $f_{b 5}$ and their amplitudes $A_{b 1}, A_{b 2}, A b_{3}, A b_{4}$ and $A b_{5}$, in Gamma range $f_{g 1}, f_{g 2}, f_{g 3}$ and their amplitudes $A_{g 1}, A_{g 2}$ and $A_{g 3}$. Similarly, brain waves and brain wave 
frequency in Alpha range $f_{a 1}$ and its amplitude $A_{a 1}$.

Hence the total brain wave energy BWE would be

$$
\begin{aligned}
\mathrm{BWE} & =C \cdot A_{b 1}{ }^{2} \cdot f_{b 1}+C \cdot A_{b 2}{ }^{2} \cdot f_{b 2}+C \cdot A_{b 3}{ }^{2} \cdot f_{g 3}+C \cdot A_{b 4}{ }^{2} \cdot f_{g 4}+C \cdot A_{b 5}{ }^{2} \cdot f_{g 5}+C \cdot A_{g 1}{ }^{2} \cdot f_{b 1}+C \cdot A_{g 2}{ }^{2} \cdot f_{b 2}+C \cdot A_{g g_{3}}{ }^{2} \cdot f_{b 3}+C \cdot A_{a 1}{ }^{2} \cdot f_{a 1} \cdot \\
& =C\left(A_{b 1}{ }^{2} \cdot f_{b 1}+A_{b 2}{ }^{2} \cdot f_{b 2}+A_{b 3}{ }^{2} \cdot f_{b 3}+A_{b 4}{ }^{2} \cdot f_{b 4}+A_{b 5}{ }^{2} \cdot f_{b 5}+A_{g 1}{ }^{2} \cdot f_{g 1}+A_{g 2}{ }^{2} \cdot f_{g 2}+A_{g 3}{ }^{2} \cdot f_{g 3}+A_{a 1}{ }^{2} \cdot f_{a 1}\right)
\end{aligned}
$$

since energy of a brain wave is proportional to the square of its amplitude.

If we can measure the brain energy and can plot its wave in frequency domain, then we can find out the value of the consciousness constant Conscire C. The brain wave energy of brain waves is so weak that it is hardly measurable at all with current tools. As technology advances, it would be possible to measure such weak signal. Then the value of consciousness constant $C$ in joules $(X)$ or in electron volt $(\mathrm{eV})(Y)$ can be determined as

$C=X$ joules or $Y \mathrm{eV}$ per Hz. 1 joule $=1 \mathrm{Kg} \mathrm{m}^{2} \mathrm{~s}^{-2}=$ $6.24 \times 10^{18} \mathrm{eV}$

\section{Unconscious State}

We are conscious as long as there is brain wave. We say that patients are made unconscious by anaesthesia for surgical operation. But the term unconscious is strictly not true. The patient has still consciousness. The patient has still heart beat and is breathing. Heart beat and breathing are controlled by lower part of brain called brainstem. Across the whole brain in anaesthetised patient, the brain wave has a low frequency around $1 \mathrm{~Hz}$ which is in low Delta range [3]. Within localized brain regions neurons are active for a few hundred milliseconds, then shut off for a few hundred milliseconds. When this area was active, it is unlikely that the other areas were not active. Hence it could not send information to other areas. This flickering of activity creates the low brain wave frequency.

Anaesthesia is given to a patient so that she does not feel any pain due to surgery. Pain starts at the source of an injury or inflammation. The spinal cord carries the pain message from its receptors all the way up to the brain, where it is received by the thalamus and sent to the cerebral cortex. Any single individual area of the brain cannot be identified as "pain center". Pain is a combination of sensory, affective and cognitive components that requires multistage processing including first-order processing in subcortical structures and higher-order processing in the cerebral cortex. Each stage and component are mediated not by one, but by a multitudinous group of regions. Anaesthesia interrupts normal firing patterns of neurons and the ability of different brain regions to communicate with each other, thus nullifying the creation of pain.

\section{Conclusion}

Many theories have been proposed to solve the mystery of consciousness, but there is no consensus to come to a definite conclusion to solve this mystery. The best and only way out of this dead-end is to accept that consciousness must be physical property of brain waves. The logic is:

- Science has proven that brain waves and brain energies exist.

- A person who is brain dead is not conscious, and brain waves and brain energies do not exist.

- Brain waves and brain energies exist only for living objects with brain.

- The nature and state of consciousness depends on the frequency of the brain waves.

\section{References}

[1] Das, T. 2017. "Consciousness as a Function of Brain Waves and Physical Constant Conscire." Neuro Quantology 15 (3).

[2] Dehaene, S. 2014. Consciousness and the Brain. New York: Penguin Group.

[3] Amthor, F. 2017. Neuroscience. Hoboken, New Jersey, USA: John Wiley\& Sons, Inc. 